\title{
Integrated metabolomic and transcriptomic analysis of the anthocyanin regulatory networks in Salvia miltiorrhiza Bge. flowers
}

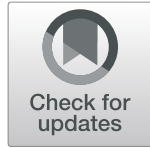

Tao Jiang ${ }^{1 \dagger}$, Meidi Zhang ${ }^{2 \dagger}$, Chunxiu Wen ${ }^{1}$, Xiaoliang Xie', Wei Tian', Saiqun Wen ${ }^{1}$, Ruike Lu ${ }^{1}$ and Lingdi Liu*

\begin{abstract}
Background: The objectives of this study were to reveal the anthocyanin biosynthesis metabolic pathway in white and purple flowers of Salvia miltiorrhiza using metabolomics and transcriptomics, to identify different anthocyanin metabolites, and to analyze the differentially expressed genes involved in anthocyanin biosynthesis.

Results: We analyzed the metabolomics and transcriptomics data of S. miltiorrhiza flowers. A total of 1994 differentially expressed genes and 84 flavonoid metabolites were identified between the white and purple flowers of S. miltiorrhiza. Integrated analysis of transcriptomics and metabolomics showed that cyanidin 3,5-O-diglucoside, malvidin 3,5-diglucoside, and cyanidin 3-O-galactoside were mainly responsible for the purple flower color of $S$. miltiorrhiza. A total of 100 unigenes encoding 10 enzymes were identified as candidate genes involved in anthocyanin biosynthesis in S. miltiorrhiza flowers. Low expression of the ANS gene decreased the anthocyanin content but enhanced the accumulation of flavonoids in S. miltiorrhiza flowers.

Conclusions: Our results provide valuable information on the anthocyanin metabolites and the candidate genes involved in the anthocyanin biosynthesis pathways in S. miltiorrhiza.
\end{abstract}

Keywords: Medicinal plant, Anthocyanin, Transcriptome, Metabolite, S. Miltiorrhiza

\section{Background}

Salvia miltiorrhiza Burge, known as "Danshen" in China, belongs to the Labiatae family and is a perennial herb from the Salvia genus. This plant has been widely cultivated in China for two thousand years. S. miltiorrhiza is an important medicinal plant, and the rhizome is used extensively for the treatment of cardiovascular and cerebrovascular diseases [1]. The extract from roots of S. miltiorrhiza is composed of many chemical components including phenolic acids, flavonoids, and polysaccharides, which are used in traditional Chinese medicine. In particular, tanshinone, a diterpene quinone extracted from $S$.

\footnotetext{
* Correspondence: nkyliulingdi@126.com

${ }^{+}$Tao Jiang and Meidi Zhang contributed equally to this work.

'Institute of Cash Crops, Hebei Academy of Agricultural and Forestry Sciences, Shijiazhuang 050051, Hebei, China

Full list of author information is available at the end of the article
}

miltiorrhiza, exhibits a wide range of pharmacological effects and may be useful in the treatment of Alzheimer's disease [2] and the prevention of osteoporosis [3].

S. miltiorrhiza Bge. f. alb is an intraspecific variety of S. miltiorrhiza Bge. that is found in Shandong Province in China [4]. Morphological differences in varieties of this species include differences in flower color, e.g., the flower color of S. miltiorrhiza Brge. is purple, while that of S. miltiorrhiza Bge. F. alba is white. Flower color is influenced by many internal and external factors, but anthocyanin content and type are among the most important factors that determine flower color. Anthocyanins that belong to the flavonoid family are important natural colorants widely distributed among leaves, flowers, fruits, and roots. Over 635 anthocyanins have been identified so far [5]. Cyanidin (Cy), delphinidin $(\mathrm{Dp})$, pelargonidin $(\mathrm{Pg})$, peonidin $(\mathrm{Pn})$, petunidin $(\mathrm{Pt})$, 
and malvidin (Mv) are six common anthocyanin pigments that are the sources of purple, blue, and red colors [6]. The biosynthesis of anthocyanin is catalyzed by a series of enzymes in the phenylpropanoid and flavonoid biosynthetic pathways $[7,8]$. The genes involved in the biosynthesis of anthocyanin have been reported in many plant species such as Arabidopsis [9], spinach [10], and alfalfa [11]. Studies have found that color mutations are often found in fruits, flowers, and leaves. Color variation can be affected by single-gene mutations in apples [12]. The expression of genes coding for enzymes and transcription factors play multiple key roles in regulating anthocyanin biosynthesis [13]. For example, high expressions of dihydroflavonol 4-reductase (DFR), anthocyanidin synthase (ANS), and anthocyanidin 3-O-glucosyltransferase (UFGT) commonly increase color accumulation in fruits [14], and the R2R3-MYB, basic helix-loop-helix (bHLH), and WD40 transcription factors can form an MBW complex to regulate the biosynthesis of anthocyanin [15-17]. The formation of pigment relies on hydroxylation, glycosylation, methoxylation, and acylation to maintain stability $[18,19]$. Some distinctive transporters including GST, the ABC transporter, MATE, and SNARE play important roles in anthocyanin transport in plants [20].

UPLC/ESI-Q TRAP-MS/MS is popular in the field of identification and analysis of plant metabolites and has the advantages of high sensitivity and throughput, fast separation, and wide coverage. So far, this technology has been widely applied to analyze the metabolites in tomato, strawberry, and asparagus [21-23]. In recent years, metabolomics integrated with transcriptomics has been widely used to investigate the biosynthesis of metabolites to reveal the biosynthesis pathways of metabolites in plants $[24,25]$. In this study, we conducted research on the regulatory networks of anthocyanin biosynthesis in flowers of S. miltiorrhiza using metabolomics and transcriptomics. We aimed to identify different anthocyanin metabolites and to analyze the differentially expressed genes (DEGs) involved in anthocyanin biosynthesis. Our results provide not only candidate genes but also valuable information for metabolic engineering of anthocyanin biosynthesis in flowers of S. miltiorrhiza.

\section{Results}

Measurement of total flavonoids and anthocyanins in flowers of $S$. miltiorrhiza

Flavonoids, especially anthocyanins, are the predominant pigment molecules in plants. In our study, the total flavonoids and anthocyanins in S. miltiorrhiza flowers were measured. The results showed that the total flavonoid content of WFSM was about $5.83 \mathrm{mg} / \mathrm{g}$ of fresh weight, which was higher than the $5.05 \mathrm{mg} / \mathrm{g}$ fresh weight of PFSM. The relative anthocyanin content of WFSM was
2.72 units/g of fresh weight, which was significantly lower than the 9.93 units/g fresh weight of PFSM (Fig. 1).

\section{Flavonoid metabolites in flowers of S. miltiorrhiza}

To compare the differential flavonoid metabolites between the white and purple flowers of S. miltiorrhiza, the data obtained from UPLC/ESI-Q TRAP-MS/MS were analyzed. In this work, a total of 84 different flavonoid metabolites were identified in flowers of $S$. miltiorrhiza (Table S2). The heatmap of metabolites was drawn by $\mathrm{R}$ software after unit variance scaling (UV), and hierarchical cluster analysis (HCA) was performed on the accumulation pattern of metabolites among different samples (Fig. 2a). The 84 flavonoid metabolites were classified into 8 categories, including 36 flavonoids, 27 flavonols, 11 anthocyanins, 3 isoflavones, 2 flavanols, 2 dihydroflavonols, 2 dihydroflavones, and 1 chalcone (Fig. 2b). So far, few studies have qualitatively or quantitatively studied flavonoids from the flowers of $S$. miltiorrhiza or their biosynthesis pathway. From our data, the skeletons of most flavonoids in flowers of S. miltiorrhiza included apigenin, quercetin, and kaempferol. Most of the flavonoids in S. miltiorrhiza flowers were Oglycosides, with only few being C-glycosides. Among the flavonoid metabolites, isorhamnetin, luteolin, isoquercitrin, quercetin, hyperin, chrysoeriol, apigenin, kaempferol, and their glycosides were abundant in flowers of $S$. miltiorrhiza. Using the identification criterion of the absolute $\log _{2} \mathrm{FC} \geq 1$ and VIP value $\geq 1,18$ flavonoid contents (21.4\% of the total), including 12 upregulated metabolites and 6 downregulated metabolites, were found to be significantly different among the 84 flavonoid metabolites in WFSM vs. PFSM. Among them, quercetin 3-O- $\beta$-D-glucoside, hyperin, hesperetin 5-O-glucoside, isoquercitrin, hesperetin 5-O-glucoside, and ladanein demonstrated significantly higher (2.48-fold to 3.11-fold) contents in WFSM vs. PFSM (Fig. 3a).

In total, 11 anthocyanins were identified in flowers of S. miltiorrhiza, including cyanidin, petunidin, peonidin, pelargonidin, delphinidin, and malvidin. Among them, the contents of cyanidin 3,5-O-diglucoside, malvidin 3,5diglucoside, and cyanidin 3-O-galactoside were significantly higher in PFSM vs. WFSM, i.e., 620.16-fold, 42.67-fold, and 2.04-fold, respectively (Fig. 3b). Cyanidin 3,5-O-diglucoside, malvidin 3,5-diglucoside, and cyanidin 3-O-galactoside could be mainly responsible for the purple flower color of S. miltiorrhiza.

Differentially expressed genes in flowers of S. miltiorrhiza To understand the molecular basis of anthocyanin biosynthesis in flowers of S. miltiorrhiza, transcriptomes were analyzed to identify differentially expressed genes in the flowers. A total of 48.95 million clean reads were produced from the flowers of $S$. miltiorrhiza. All clean 

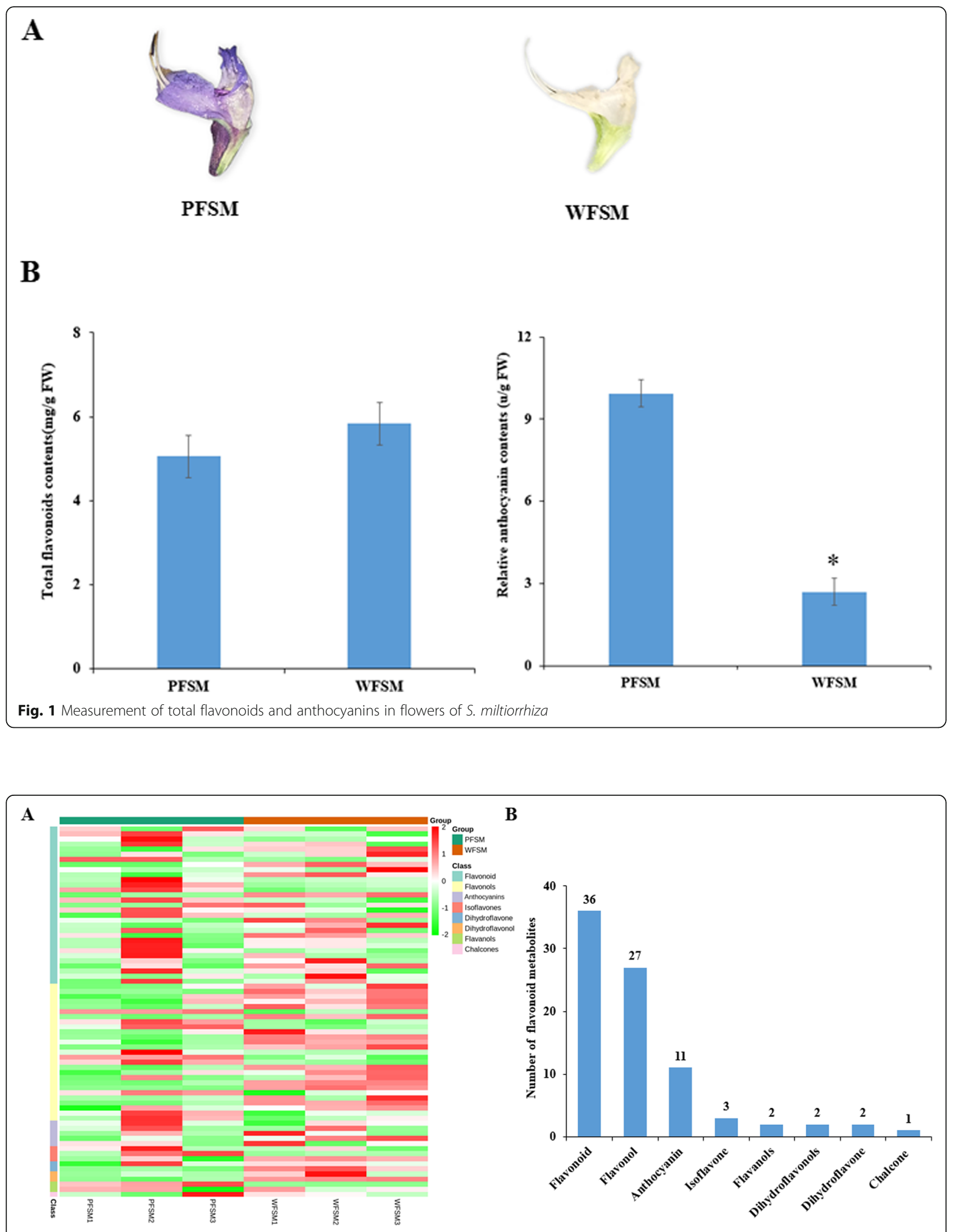

B

Fig. 2 Flavonoid metabolites in PFSM and WFSM 
A
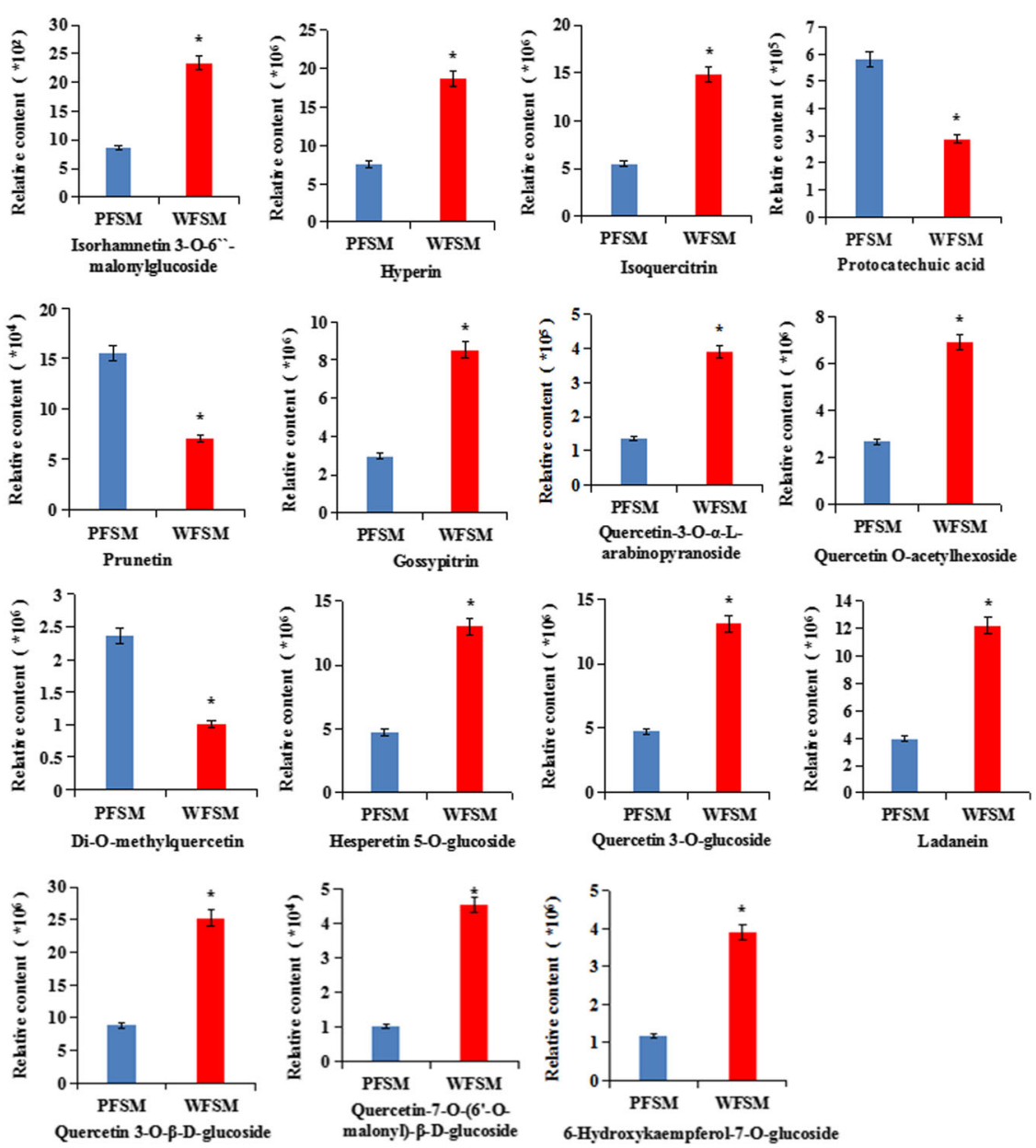

B
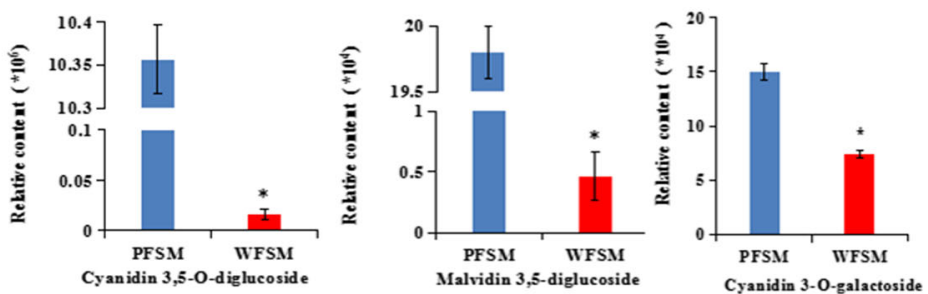

Fig. 3 Relative contents of flavonoid and anthocyanin metabolites in PFSM and WFSM

reads were searched in the KEGG, NR, Swiss-Prot, KOG, GO, and Pfam databases with the BLASTX program for functional annotations. A total of 28,539 unigenes were annotated functionally based on the above-mentioned databases. With the filter criteria $\left|\log _{2} \mathrm{FC}\right| \geq 1$ and $\mathrm{FDR}<0.05,1994$ differentially expressed genes (DEGs) were identified in the flowers of S. miltiorrhiza, including 1173 upregulated genes and 821 downregulated genes in WFSM vs. PFSM (Fig. 4a and Table S3). In GO enrichment analysis of DEGs, 1304 DEGs out of 1994 were involved in three major GO categories, i.e., biological processes, cellular components, and molecular functions, and 19 anthocyanin biosynthetic processes and 33 flavonoid biosynthetic processes were identified in the biological process category (Fig. 4b).

To understand their biological functions and gene interactions, 807 of 1994 DEGs were annotated by the KEGG database. KEGG metabolic pathway enrichment analysis using a Q-value $<0.05$ showed the DEGs were enriched in many metabolic processes that included flavonoid and anthocyanin biosynthesis pathways (Fig. 5a). Among these 1994 DEGs, we found 30 glycosyltransferase genes, 25 methyltransferase genes, and 1 acyltransferase gene that 


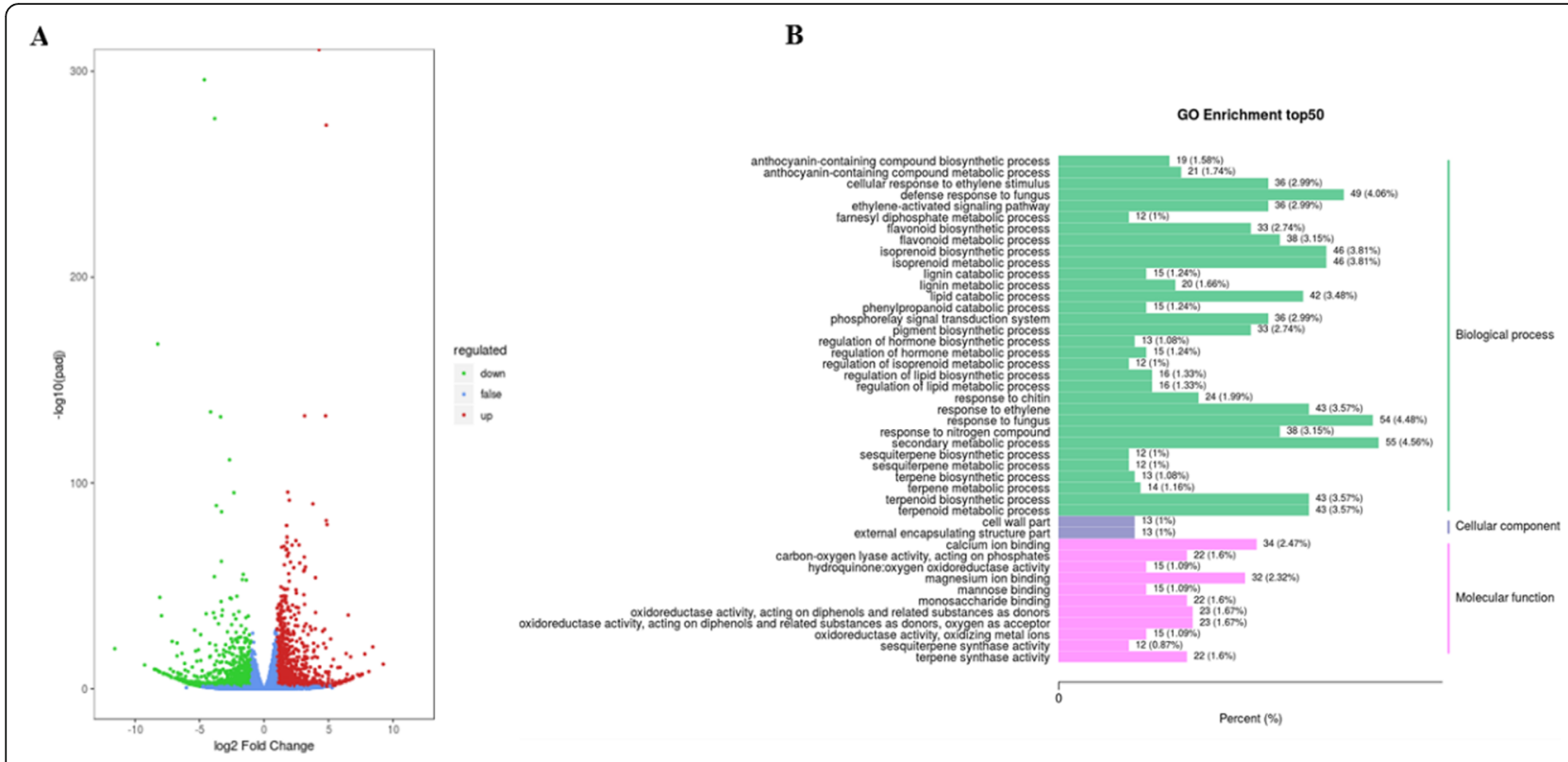

Fig. 4 Transcriptome analysis of genes in PFSM and WFSM

catalyzed the synthesis of different types of flavonoids and anthocyanins. In further analysis of the DEGs, we also detected 6 GST genes, 12 ABC transporter genes, 9 MATE genes, and 7 SNARE genes, which might play important roles in transporting anthocyanin to plant vacuoles (Fig. 5b).

\section{Combined transcriptome and metabolome analysis} revealed the biosynthesis of anthocyanin in flowers of $S$.

\section{miltiorrhiza}

We combined the analysis of transcriptomic and metabolomic data to understand the pigment biosynthesis pathway in flowers of S. miltiorrhiza. The result demonstrated that a large number of flavonoids and anthocyanins were detected in flowers of S. miltiorrhiza. Among them, the flavonoid (catalyzed by CHS, CHI, or F3'H) content was high, whereas anthocyanin (catalyzed by ANS) content was lower in WFSM vs. PFSM. To understand this finding, the transcriptome of WFSM was compared with that of PFSM. Analysis of unigenes involved in flavonoids, especially the anthocyanin biosynthesis pathway, was performed to mine the key genes in the metabolism of purple and white flowers of S. miltiorrhiza. In total, 100 unigenes that encoded 10 enzymes in the flavonoid and anthocyanin biosynthesis pathways were studied (Table 1). Twenty-one key unigenes had different expression levels; there were 19 upregulated and 2 downregulated unigenes

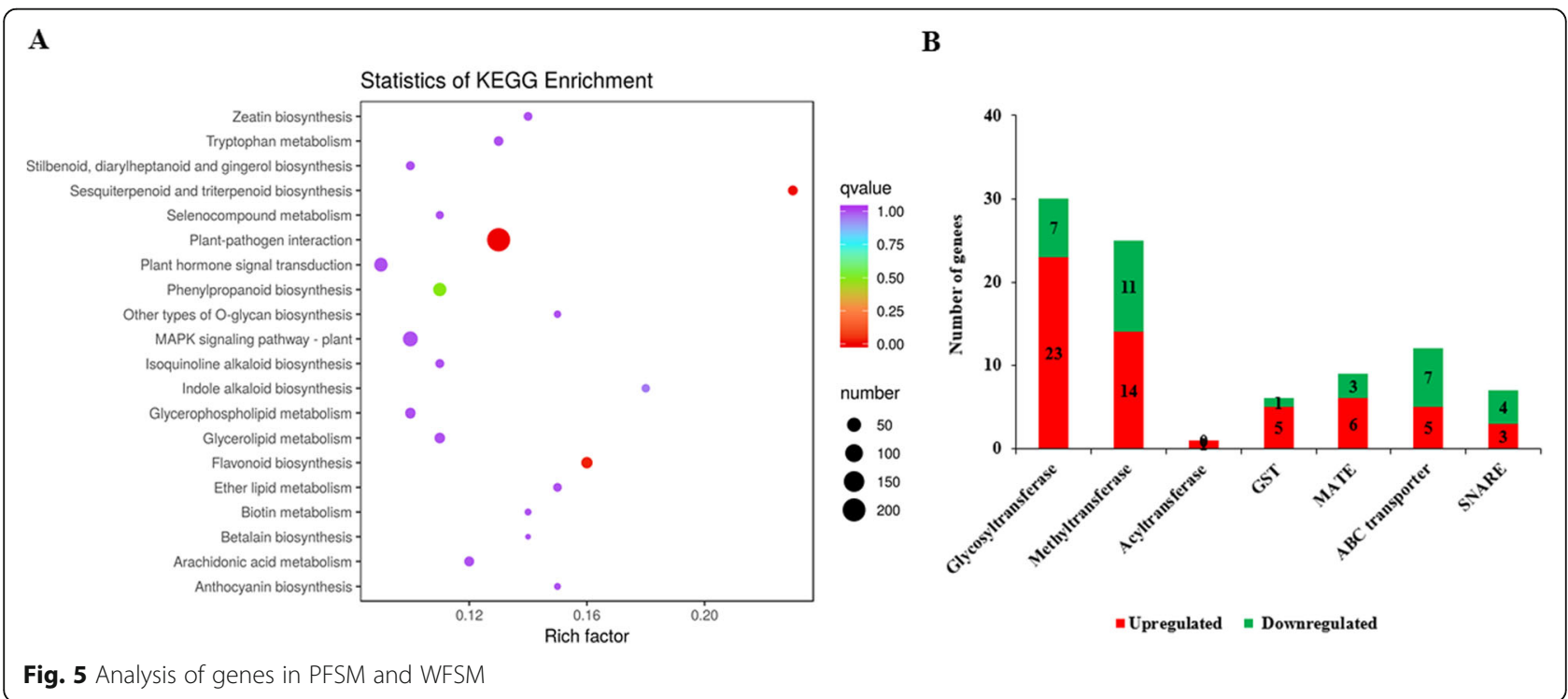


Table 1 Candidate genes related to anthocyanin biosynthesis in flowers of S. miltiorrhiza

\begin{tabular}{|c|c|c|c|c|}
\hline Gene & Enzyme & No. Alla & No. Up & No. Downc \\
\hline$\overline{\mathrm{CHS}}$ & Chalcone synthase & 9 & 7 & 0 \\
\hline $\mathrm{CH}$ & Chalcone isomerase & 11 & 1 & 0 \\
\hline $\mathrm{F} 3 \mathrm{H}$ & Flavonone 3-hydroxylase & 32 & 3 & 0 \\
\hline $\mathrm{F}^{\prime} \mathrm{H}$ & Flavonoid 3'-monooxygenase & 15 & 1 & 0 \\
\hline $\mathrm{F}^{\prime} \mathrm{S}^{\prime} \mathrm{H}$ & Flavonoid 3',5'-hydroxylase & 4 & 1 & 0 \\
\hline FNS ॥ & Flavone synthase II & 1 & 1 & 0 \\
\hline DFR & Dihydroflavonol 4-reductase & 5 & 3 & 0 \\
\hline ANS & Anthocyanidin synthase & 7 & 0 & 2 \\
\hline UAGT & Anthocyanidin 3-O-glucosyltransferase & 15 & 2 & 0 \\
\hline UGT75 & Anthocyanidin 3-O-glucoside 5-O-glucosyltransferase & 1 & 0 & 0 \\
\hline
\end{tabular}

No. All ${ }^{\mathrm{a}}$, the total number of genes

No. Up ${ }^{b}$, the number of upregulated genes

No. Down', the number of downregulated genes

in WFSM vs. PFSM. The core genes in the anthocyanin pathway were analyzed in detail, and the results showed the early genes (CHS, CHI, etc.) or late genes (DFR, UFGT, etc.) had higher expression levels except the ANS gene in white compared with purple flowers. Among the DEGs, 7 CHS, 1 CHI, 3 F3H, 1 F3'H, 1 F3'5'H, 1 FNS II, 3 DFR, and 2 UAGT genes were upregulated by 1.033- to 3.13fold $\left(\log _{2} \mathrm{FC}\right.$ ), whereas 2 ANS genes (SMil_00006040 and SMil_00029434) were downregulated by - 1.304- to 4.631-fold (Fig. 6 and Table S4). These DEGs affected the biosynthesis of anthocyanin in flowers of S. miltiorrhiza.

The key genes responsible for anthocyanin biosynthesis in flowers of S. miltiorrhiza

The 2 differentially expressed ANS genes were further assigned to the ANS family in the NCBI database. Blast analysis indicated that the SMil_00029434 protein had 99.41\% sequence identity to SmANS (S. miltiorrhiza, AWX67417.1), while the SMil_00006040 protein exhibited $81.19,71.01,69.3$, and $68.59 \%$ amino acid identity to SiANS (Sesamum indicum, XP_020551541.1), OeANS (Olea europaea var. sylvestris, XP_022887681.1), DhANS (Dorcoceras hygrometricum, KZV49046.1), and CaSnRK1 (Coffea arabica, XP_027087242.1), respectively (Fig. 7a). Phylogenetic analysis revealed that the SMil_00006040 protein had a close relationship with the ANS protein of Sesamum indicum and Olea europaea var. sylvestris (Fig. 7b). The sequence of the SMil_00006040 gene was not reported in the NCBI database. We believe the SMil_00006040 gene is a new ANS gene in S. miltiorrhiza.

\section{Transcription factors of anthocyanin biosynthesis in flowers of S. miltiorrhiza}

Transcription factors participate in anthocyanin biosynthesis processes by regulating the gene expression in plants. In our data, 90 transcription factors with different expression levels were related to anthocyanin biosynthesis
(Table 2). Among these transcription factors, 14 genes were annotated as MYB-related genes, including MYB36, MYB44, MYB113, and other MYB-dominant proteins. Nine genes encoding bHLH, 1 WD40 gene, and other transcription factors were also found in this study. These transcription factors may contribute to anthocyanin metabolite biosynthesis in the flowers of S. miltiorrhiza.

\section{QRT-PCR of the transcriptomic data}

To verify the credibility of the transcriptome information, we further selected 12 DEGs to validate the sequencing results. The qRT-PCR results showed that 11 genes showed higher expression levels and 1 gene had a lower expression in WFSM than in PFSM; our qRT-PCR results were consistent with those obtained with the RNA-Seq method (Fig. 8).

\section{Discussion}

Anthocyanin identification in the flowers of S. miltiorrhiza Anthocyanins are secondary metabolites in plants such as vegetables, fruits, and medical plants and have many diverse biological functions including antioxidation, regulation of defense responses, and protection against ultraviolet radiation [26]. Anthocyanins are synthesized at the end of the phenylpropanoid metabolic pathway, and the precursors of anthocyanin biosynthesis are malonyl-CoA and coumaroyl-CoA. Most anthocyanins are synthesized through $\mathrm{CHS}$ and $\mathrm{CHI}$ condensation; F3H, F3'H, or F3'5'H oxidation; or DFR and ANS/ LDOX catalysis [27-29]. In our study, in order to elucidate anthocyanin biosynthesis in white and purple flowers of S. miltiorrhiza, metabolomic and transcriptomic pathways were analyzed in S. miltiorrhiza flowers. A total of 73 flavonoids and 11 anthocyanins were identified in flowers of $S$. miltiorrhiza by UPLC/ESI-Q TRAP-MS/MS (Table S2). We found that the total 


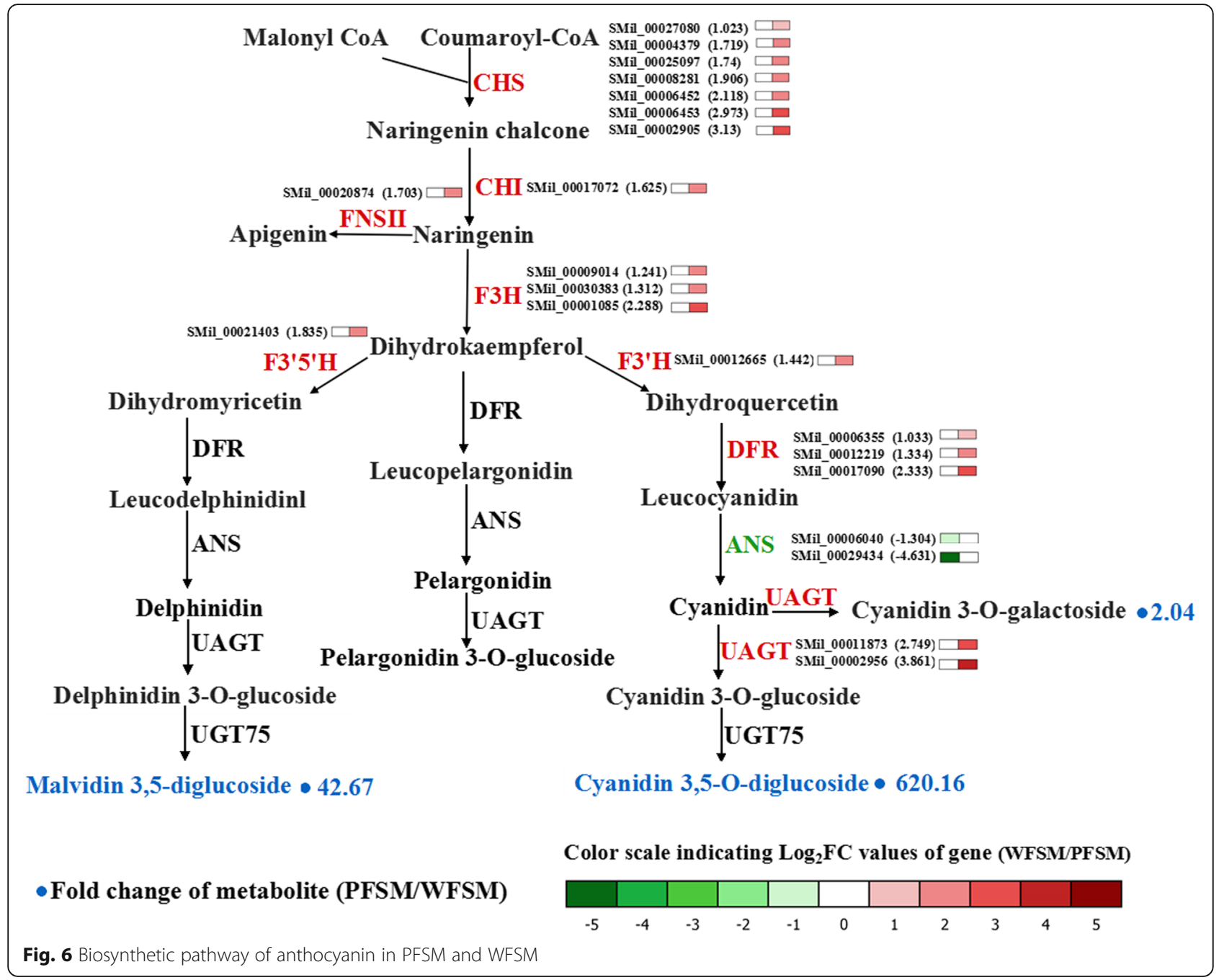

flavonoid content was higher and the anthocyanin content lower in WFSM vs. PFSM (Figs. 1 and 3). Cyanidin 3,5-O-diglucoside, cyanidin 3-O-galactoside, and malvidin 3,5-diglucoside were significantly different anthocyanin metabolites between WFSM and PFSM. Among the above anthocyanins, the cyanidin 3,5-O-diglucoside content was 620.16-fold higher in PFSM vs. WFSM (Fig. 3b), and the content of cyanidin 3,5-O-diglucoside was also higher than that of other anthocyanins in PFSM. This result suggests that cyanidin 3,5-O-diglucoside is the main anthocyanin in PFSM.

Interestingly, we found that cyanidin, petunidin, peonidin, pelargonidin, delphinidin, and malvidin were present not only in PFSM but also in WFSM (Table S2). Quintana et al. [30] suggested that there is a mutation in the early stage of the anthocyanin pathway, leading to no anthocyanidins in white flowers of Anagallis monellin. Duan et al. [11] reported that malvidin and petunidin were detected in purple alfalfa flowers, whereas no color anthocyanidins occurred in cream alfalfa flowers. In this study, we found that 6 types of common anthocyanin pigments were present simultaneously in white flowers of $S$. miltiorrhiza. It is possible that the determinants of flower color are involved in the number of pigments, metal ions, or the different molecular conformations of anthocyanin. These pigments are present in small quantities in white flowers of S. miltiorrhiza, suggesting that complete anthocyanin metabolic pathways induce anthocyanin biosynthesis in the white flowers of this species.

The genes involved in anthocyanin biosynthesis in flowers of S. miltiorrhiza

Transcriptome analysis of the flowers of S. miltiorrhiza identified unigenes involved in the anthocyanin biosynthesis process and showed differentially expressed genes. In the anthocyanin biosynthesis pathway, the expression levels of genes encoding CHS, CHI, FNS II, F3H, F3'H, F3 ${ }^{`}{ }^{\prime} \mathrm{H}$, DFR, and UAGT were higher in WFSM than in PFSM (Fig. 6), which resulted in a higher flavonoid content in the white flowers of $S$. miltiorrhiza. ANS is a key 


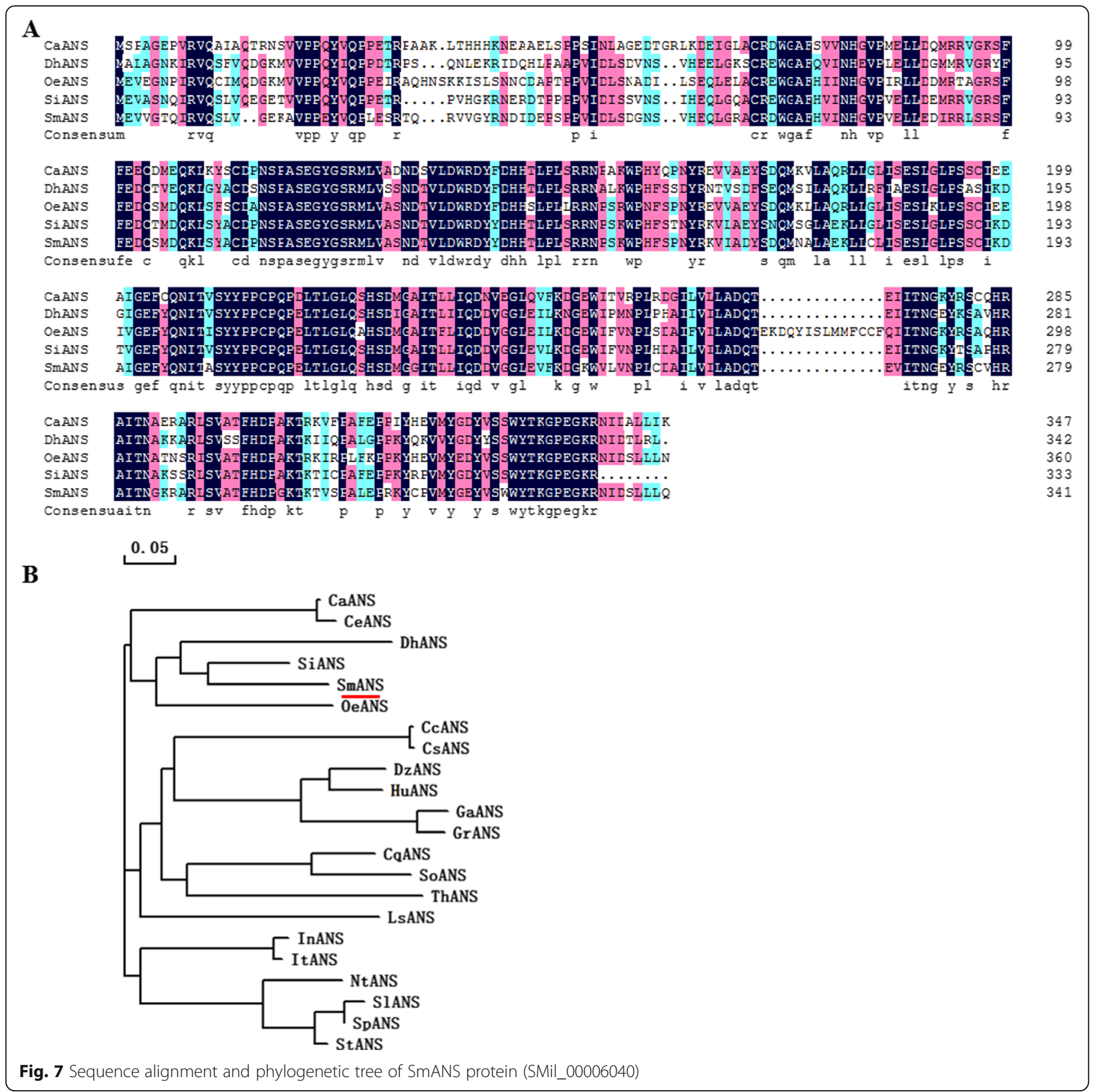

enzyme of the anthocyanin pathway that plays an important role in the conversion of leucoanthocyanidins to anthocyanidins [31]. In this study, we detected two differentially expressed ANS genes (SMil_00006040 and SMil_00029434) that were downregulated in WFSM vs. PFSM (Table S4). Blast analysis indicated that the SMil_ 00006040 gene is a new ANS gene in S. miltiorrhiza. The low expression level of the ANS gene may have inhibited the formation of anthocyanin, leading to the formation of white flowers. Based on the metabolomic and transcriptomic data, we speculated that the low expression of the ANS gene decreased the anthocyanin content but enhanced the accumulation of flavonoids in flowers of S. miltiorrhiza.

The function of the ANS gene in flowers of S. miltiorrhiza ANS is one of the four dioxygenases in the anthocyanin biosynthetic pathway that catalyzes the formation of anthocyanidins. Some studies have shown that the absence of ANS and DFR genes in the anthocyanin biosynthesis pathway would lead to the loss of pigmentation [32, 33]. The white-fruited Duchesnea indica (Rosaceae) phenotype is related to the downregulation of the ANS gene [34]. The suppressed expression of the ANS gene 
Table 2 Transcription factors of anthocyanin biosynthesis in flowers of S. miltiorrhiza

\begin{tabular}{|c|c|c|c|c|}
\hline Gene & Enzyme & No. All ${ }^{\mathbf{a}}$ DEGs & No. Up $p^{\mathbf{b}}$ DEGs & No. Down ${ }^{c}$ DEGs \\
\hline$\overline{M Y B}$ & MYB TF & 14 & 10 & 4 \\
\hline bHLH & Basic helix-loop-helix protein & 9 & 6 & 3 \\
\hline WD40 & WD40 repeat protein & 1 & 0 & 1 \\
\hline AP2/ERF & Ethylene-responsive TF & 33 & 30 & 3 \\
\hline WRKY & WRKY DNA-binding protein & 4 & 4 & 0 \\
\hline MADs & MADS-box TFs & 5 & 4 & 1 \\
\hline NAC & NAC domain containing protein & 4 & 2 & 2 \\
\hline Zinc-finger & Zinc finger protein & 12 & 10 & 2 \\
\hline bZIP & Basic leucine zipper & 5 & 3 & 2 \\
\hline LBD & Lateral organ boundary domain & 3 & 2 & 1 \\
\hline
\end{tabular}

No. All ${ }^{\mathrm{a}} \mathrm{DEGs}$, the total number of DEGs

No. Up ${ }^{\mathrm{b}}$ DEGs, the number of upregulated DEGs

No. Down ${ }^{\mathrm{C}} \mathrm{DEGs}$, the number of downregulated DEGs
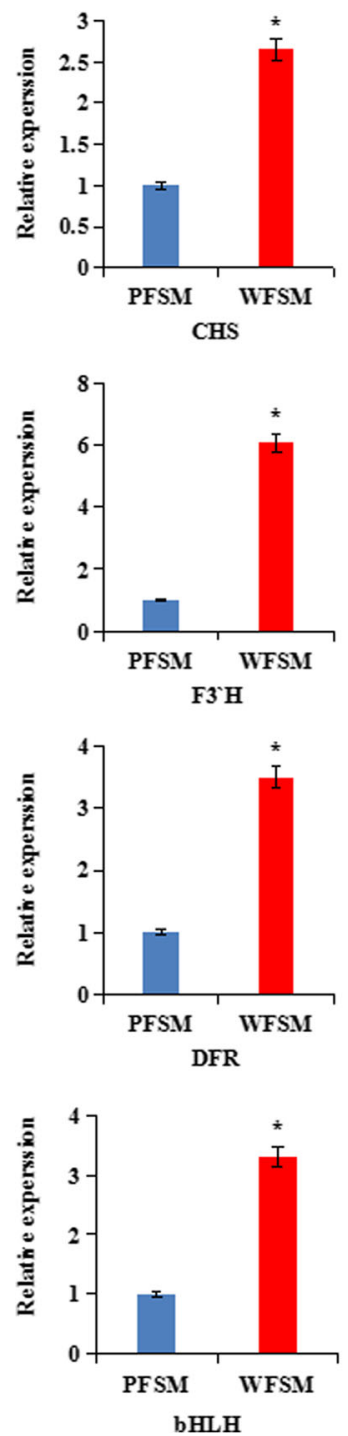
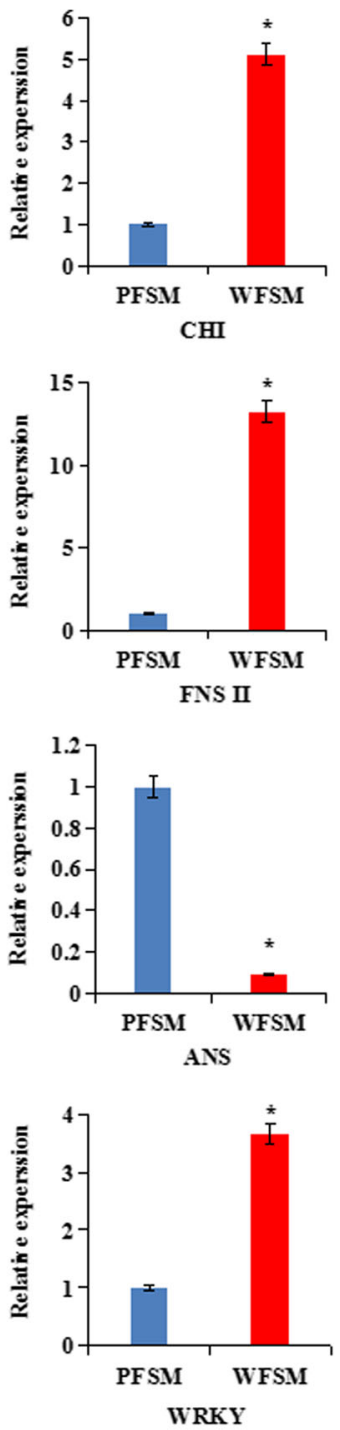
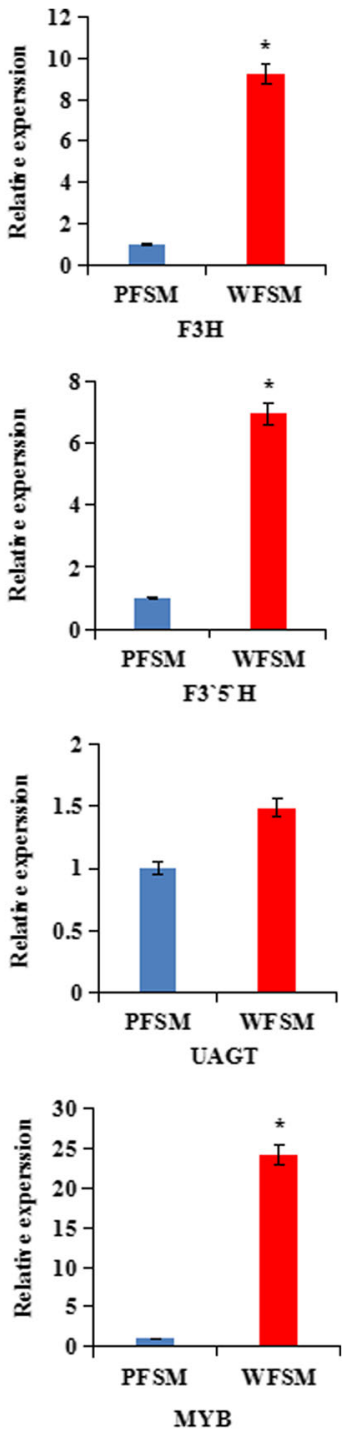

Fig. 8 Relative Expression levels of 12 genes involved in the anthocyanin biosynthetic pathway by qRT-PCR analysis 
leads to a lack of anthocyanins in Caryophyllales [35]. Li et al. [36] reported that overexpression of SmANS increased anthocyanin content but reduced the biosynthesis of salvianolic acid B in S. miltiorrhiza. Whiteflowered $S$. miltiorrhiza is a variety of the S. miltiorrhiza species. Our analysis suggested that the white-flowered character was due to the low expression of the ANS gene. Next in a future study, we will overexpress ANS genes (SMil_00006040 and SMil_00029434) to research their functions in white-flowered S. miltiorrhiza (Fig. 7).

\section{Transcription factors related to anthocyanin biosynthesis}

Anthocyanin biosynthesis is mostly regulated by transcription factors at the transcription level. To date, transcription factors of Myb, bHLH, WD40, zinc finger, MADs, and WRKY proteins have been found to regulate anthocyanin biosynthesis [37, 38]. Among them, MYB transcription factors play a key role in the regulation of anthocyanin biosynthesis. In particular, MYB75/PAP1 is a master regulator controlling anthocyanin biosynthesis in Arabidopsis [39]. In the anthocyanin biosynthetic pathway, anthocyanin biosynthesis is controlled by the MYB-bHLH-WD40 (MBW) complex in plant species such as Arabidopsis [26], maize [40], and cauliflower [41]. In addition to MYBs, the zinc-finger transcription factor (AtZAT6) is also involved in anthocyanin synthesis through direct binding to the promoters of several genes in Arabidopsis [42]. The bHLH transcription factors have been proved to positively regulate the biosynthesis of anthocyanin in Arabidopsis [43]. MdWRKY11 can increase the expression of F3H, FLS, DFR, ANS, and UFGT to promote anthocyanin accumulation in apples [44]. The IbMADS10 gene regulates anthocyanin biosynthesis to increase the accumulation of anthocyanin pigments in sweet potato [45]. In our study, we analyzed the transcriptome data and found that 90 important transcription factors including MYB, AP2/ERFs, WRKY, bHLH, MADs, WD40, zinc-finger, NACs, bZIP, and LBD showed significantly different expression levels between WFSM and PFSM (Table 2). We speculated that these differentially expressed transcription factors may be candidate regulators of anthocyanin biosynthesis in $S$. miltiorrhiza flowers.

\section{Conclusions}

We used metabolomics and transcriptomics to reveal the anthocyanin biosynthesis metabolic pathway. A total of 1994 differentially expressed genes and 84 different flavonoid metabolites were identified in two varieties of S. miltiorrhiza. In an analysis of the anthocyanin biosynthesis pathway, we identified 100 unigenes encoding 10 enzymes that are involved in anthocyanin biosynthesis in flowers of S. miltiorrhiza. Integrated analysis of transcriptomic and metabolomic data showed that the low expression of the ANS gene decreased the anthocyanin content but enhanced the accumulation of flavonoids in white flowers of S. miltiorrhiza. Cyanidin 3,5-O-diglucoside, malvidin 3,5-diglucoside, and cyanidin 3-O-galactoside are mainly responsible for the purple flower color of $S$. miltiorrhiza. Our results provide valuable information on the anthocyanin metabolites and the candidate genes involved in the anthocyanin biosynthesis pathways in S. miltiorrhiza.

\section{Methods}

Plant materials

White-flowered S. miltiorrhiza (WFSM) and purpleflowered S. miltiorrhiza (PFSM) were grown at the $S$. miltiorrhiza germplasm resource center at the Institute of Cash Crops, Hebei Academy of Agricultural and Forestry Sciences, China. Fresh flowers were collected from healthy plants in August 2019. All materials were frozen in liquid nitrogen and stored at $-80^{\circ} \mathrm{C}$ for RNA and metabolite extraction. All experiments were performed in three biological replicates in this study.

\section{Measurement of total flavonoid content}

Approximately $2.5 \mathrm{~g}$ of $S$. miltiorrhiza flowers was used to measure the total flavonoid content by the aluminum nitrate colorimetric method. In brief, $0.5 \mathrm{~mL}$ of crude flower extract was mixed with $5.5 \mathrm{~mL}$ of $50 \%$ ethanol and $1 \mathrm{~mL}$ of $5 \% \mathrm{NaNO}_{2}$ solution. Then, $1 \mathrm{~mL}$ of $10 \% \mathrm{~A} 1\left(\mathrm{NO}_{3}\right)_{3}$ solution was added after $6 \mathrm{~min}$ of incubation, and the mixture was incubated for another $6 \mathrm{~min}$. Subsequently, $10 \mathrm{~mL}$ of $4 \% \mathrm{NaOH}$ solution and $7 \mathrm{~mL}$ of $50 \%$ ethanol were added. The final volume of the mixture was $25 \mathrm{~mL}$. The mixture stood for $15 \mathrm{~min}$, and then the absorbance was measured at a wavelength of $506 \mathrm{~nm}$ by an ultraviolet spectrophotometer (V-5100B, METASH, Shanghai, China). Rutin was used as a standard solution to prepare a calibration curve, and the results were expressed as rutin equivalent on a fresh weight basis [46].

\section{Measurement of relative anthocyanin content}

S. miltiorrhiza flowers $(0.1 \mathrm{~g})$ were ground with $1 \mathrm{~mL}$ of methanol $(0.1 \% \mathrm{HCl})$ and were washed twice into $10 \mathrm{~mL}$ centrifuge tubes. The final volume of the samples was 5 $\mathrm{mL}$ (including methanol $(0.1 \% \mathrm{HCl})$ ). The tissue homogenates were oscillated for $30 \mathrm{~s}$ and centrifuged at $4{ }^{\circ} \mathrm{C}$ and $12,000 \mathrm{~g}$ for $10 \mathrm{~min}$, and the absorbance of the supernatants was measured at a wavelength of $530 \mathrm{~nm}$ using an ultraviolet spectrophotometer (V-5100B, METASH). The relative anthocyanin concentration was calculated using the following formula: $\mathrm{Q}=\mathrm{V} * \mathrm{~A} 530$ / $\mathrm{M}$ (units/g FW), where $\mathrm{V}$ represents the volume of the solution, and $M$ represents the weight of the sample. Methanol $(0.1 \% \mathrm{HCl})$ was used as a blank control [47]. 


\section{Metabolite extraction}

Freeze-dried flowers were crushed using a mixer mill (MM 400, Verder Retsch, Shanghai, China) with a zirconia bead for $1.5 \mathrm{~min}$ at a frequency of $30 \mathrm{~Hz}$. Then, $100 \mathrm{mg}$ powder was weighed and extracted overnight at $4{ }^{\circ} \mathrm{C}$ with $1.0 \mathrm{~mL}$ $70 \%$ methanol aqueous solution $(\mathrm{V} / \mathrm{V}=70 \%)$. Following centrifugation at $10,000 \mathrm{~g}$ for $10 \mathrm{~min}$, the extracts were absorbed by a CNWBOND Carbon-GCB SPE cartridge (250 mg, $3 \mathrm{~mL}$; ANPEL, Shanghai, China, www.anpel.com. $\mathrm{cn} / \mathrm{cnw})$ and filtered through a $0.22-\mu \mathrm{m}$ microfiltration membrane (SCAA-104; ANPEL, Shanghai, China, http:// www.anpel.com.cn/) before UPLC-MS/MS analysis.

\section{Ultra-performance liquid chromatography (UPLC) conditions}

A UPLC-ESI-MS/MS system (UPLC, Shim-pack UFLC SHIMADZU CBM30A system, Shanghai, China, www. shimadzu.com.cn/) was used to analyze the sample extracts. The UPLC analysis was performed under the following conditions, UPLC: column, Waters (Shanghai, China) ACQUITY UPLC HSS T3 C18 $(1.8 \mu \mathrm{m}, 2.1$ $\mathrm{mm} * 100 \mathrm{~mm})$; solvent system, water $(0.04 \%$ acetic acid): acetonitrile $(0.04 \%$ acetic acid); gradient program, 95:5 $\mathrm{V} / \mathrm{V}$ at $0 \mathrm{~min}, 5: 95 \mathrm{~V} / \mathrm{V}$ at $11.0 \mathrm{~min}, 5: 95 \mathrm{~V} / \mathrm{V}$ at 12.0 $\mathrm{min}, 95: 5 \mathrm{~V} / \mathrm{V}$ at $12.1 \mathrm{~min}, 95: 5 \mathrm{~V} / \mathrm{V}$ at $15.0 \mathrm{~min}$; flow rate, $0.40 \mathrm{~mL} / \mathrm{min}$; temperature, $40^{\circ} \mathrm{C}$; injection volume: $2 \mu \mathrm{L}$. The effluent was alternatively connected to an ESItriple quadrupole-linear ion trap (Q TRAP)-MS.

\section{ESI-q trap-MS/MS}

Linear ion hydrazine-flight time (LIT) and triple quadrupole (QQQ) scans were conducted on a triple Q TRAP, API 6500 Q TRAP LC/MS/MS system (Applied Biosystems, Shanghai, China) equipped with an ESI turbo ionspray interface, operating in positive ion mode and negative ion mode. The system was controlled by Analyst 1.6 software (AB Sciex, Shanghai, China). The ESI source was set with the following parameters: ion source, turbo spray; source temperature $500^{\circ} \mathrm{C}$; ion spray voltage (IS) $5500 \mathrm{~V}$. The ion source gas I (GSI), gas II (GSII), and curtain gas (CUR) were set at $55.0 \mathrm{psi}, 60.0 \mathrm{psi}$, and $25.0 \mathrm{psi}$, respectively; the collision gas (CAD) was high. Instrument tuning and mass calibration were performed with 10 and $100 \mu \mathrm{mol} / \mathrm{L}$ polypropylene glycol solutions in QQQ and LIT modes, respectively. QQQ scans were acquired as multiple reaction monitoring (MRM) experiments with collision gas (nitrogen) set to $5 \mathrm{psi}$. Declustering potential (DP) collision energy (CE) measurements for individual MRM transitions were completed with further DP and CE optimization. A specific set of MRM transitions was monitored for each period according to the metabolites eluted within the period.
Identification and quantitative analysis of metabolites

Base on the stepwise MIM-EPI (multiple ion monitoringenhanced product ions) to build the commercially available standard Metabolites Database (Metware Biotechnology Co., Ltd. Wuhan, China) and the public metabolite databases such as MassBank, KNAPSAcK, HMDB, MoToDB, and METLIN, qualitative analysis of the metabolite data was performed. The quantitative analysis of metabolites used multiple reaction monitoring [48, 49]. Unsupervised PCA (principal component analysis), HCA (hierarchical cluster analysis), and OPLS-DA (partial least-squares discriminant analysis) were performed by the statistics function prcomp within $\mathrm{R}$ (www.r-project.org). Significantly different metabolites between groups were determined by VIP $\geq 1$ and an absolute $\log _{2}$ FC (fold change) $\geq 1$.

\section{RNA extraction and Illumina sequencing}

Total RNA was extracted from frozen flowers using the RNAprep Pure Plant Kit (Tiangen Biotech, Beijing, China). RNA degradation and contamination were monitored on $1.2 \%$ agarose gels. The purified RNA concentrations were quantified using a NanoDropTM 2000 spectrophotometer (Thermo Scientific, Shanghai, China). The quality of the total RNA was examined using an Agilent 2100 Bioanalyzer (Agilent Technologies, Santa Clara, CA, USA). Poly (A) mRNA was enriched from the total RNA using Oligo (dT) magnetic beads. Poly (A) mRNA was subsequently fragmented by an RNA fragmentation kit (Ambion, Austin, TX, USA). The fragmented RNA was transcribed into first-strand cDNA using reverse transcriptase and random hexamer primers. Second-strand cDNA was synthesized using DNA polymerase I and RNase H (Invitrogen, Carlsbad, CA, USA). After end repair and the addition of a poly (A) tail, suitable length fragments were isolated and connected to the sequencing adaptors. The fragments were sequenced on an Illumina HiSeq 2500 platform.

\section{RNA sequencing (RNA-seq) data analysis and annotation}

To acquire high-quality reads, the raw reads in fastq format were processed through in-house Perl scripts. Clean reads were obtained from raw data by removing adaptor sequences, low-quality reads, and reads containing ployN. All downstream analyses were based on clean, highquality data. Gene function was annotated using the following: the Kyoto Encyclopedia of Gene and Genome (KEGG) pathway database, the NCBI non-redundant $(\mathrm{Nr})$ database, the Swiss-Prot protein database, the euKaryotic Clusters of Orthologous Groups (KOG) database, the Gene Ontology (GO) database, and the Pfam database.

The levels of gene expression were estimated by RSEM (version 1.2.26) [50]. Analysis of the differentially expressed genes of the two groups was performed with 
the DESeq R package (1.10.1). DESeq provides statistical routines for determining differentially expressed genes using a model based on the negative binomial distribution. The results of all statistical tests were corrected by multiple tests using the Benjamini and Hochberg false discovery rate. Genes were determined to be significantly differentially expressed at an adjusted $P$-value of $<0.05$ according to DESeq. GO enrichment analysis of the differentially expressed genes was implemented by the topGO $\mathrm{R}$ package based on the Kolmogorov-Smirnov test. Pathway analysis elucidated significant pathways of differentially expressed genes according to the KEGG database (http://www.genome.jp/kegg/) [51]. We tested the statistical enrichment of differentially expressed genes in KEGG pathways using KOBAS software [52].

\section{QRT-PCR expression analysis of genes involved in anthocyanin biosynthesis}

Total RNA of $S$. miltiorrhiza flowers was reversetranscribed according to the Quantscript Reverse Transcriptase Kit (Tiangen Biotech, Beijing, China). cDNA was used as a template to measure gene expression. The specific primers for genes involved in anthocyanin biosynthesis and the S. miltiorrhiza actin gene (internal control) are listed in Table S1. A quantitative real-time polymerase chain reaction (qRT-PCR) was conducted by a real-time PCR ABI Prism 7500 system (software for 7500 and 7500 Fast RealTime PCR Systems, V2.0.1, Foster City, CA, USA) using SYBR $^{\circ}$ Premix Ex Taq ${ }^{\mathrm{Tm}}$ II (TaKaRa Code No. RR820A, http://www.takarabiomed.com.cn). The comparative CT method (2- ${ }^{\Delta \Delta C T}$ method) was used to quantify gene expression [53].

\section{Statistical analysis}

Statistical analysis was performed using Excel 2010 software (Microsoft Office, USA). Data are presented as means \pm standard deviations (SD). The levels of statistical significance were analyzed by the least significant difference $(p<0.05)$.

\section{Supplementary information}

Supplementary information accompanies this paper at https://doi.org/10. 1186/s12870-020-02553-7.

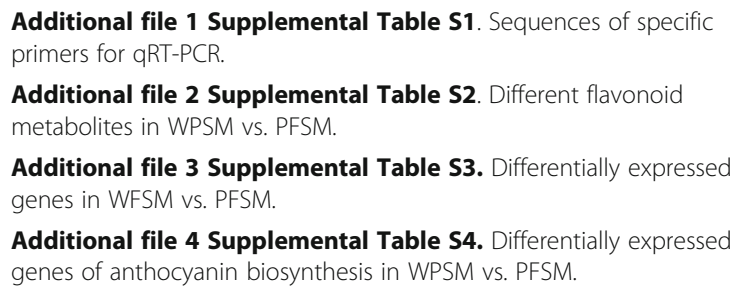

Additional file $\mathbf{3}$ Supplemental Table S3. Differentially expressed genes in WFSM vs. PFSM.

Additional file 4 Supplemental Table S4. Differentially expressed genes of anthocyanin biosynthesis in WPSM vs. PFSM.

\section{Abbreviations}

Cy: Cyanidin; Dp: Delphinidin; Pg: Pelargonidin; Pn: Peonidin; Pt: Petunidin; Mv: Malvidin; DFR: Dihydroflavonol 4-reductase; ANS: Anthocyanidin synthase; UFGT: Anthocyanidin 3-O-glucosyltransferase; CHS: Chalcone synthase; CHI: Chalcone isomerase; F3H: Flavonone 3-hydroxylase; F3' $\mathrm{H}$ : Flavonoid 3'-monooxygenase; F3'5'H: Flavonoid 3',5'-hydroxylase; FNS II: Flavone synthase II; UGT75: Anthocyanidin 3-O-glucoside 5-Oglucosyltransferase; bHLH: Basic helix-loop-helix; DEGs: Differentially expressed genes; UPLC: Ultra-performance liquid chromatography; LIT: Linear ion hydrazine-flight time; QQQ: Triple quadrupole; GSI: Gas I; GSII: Gas II; CUR: Curtain gas; CAD: Collision gas; MRM: Multiple reaction monitoring; DP: Declustering potential; CE: Collision energy; MIM-EPI: Multiple ion monitoring-enhanced product ions; PCA: Principal component analysis; HCA : Hierarchical cluster analysis; OPLS-DA: Partial least-squares discriminant analysis; KEGG: Kyoto Encyclopedia of Gene and Genome; Nr: NCBI nonredundant; KOG: EuKaryotic Clusters of Orthologous Groups; GO: Gene Ontology; qRT-PCR: Quantitative real-time polymerase chain reaction; SD: Standard deviations; UV: Unit variance scaling

\section{Acknowledgements}

We thank LetPub (www.letpub.com) for its linguistic assistance during the preparation of this manuscript.

\section{Authors' contributions}

LD and TJ conceived and designed the experiments. $X \mathrm{~L}, \mathrm{CX}$, and SQ performed the experiments. MD, WT, and RK analyzed the data. TJ and MD wrote the paper. The authors read and approved the final version of the paper.

\section{Funding}

This work was supported by the Evaluation and Application of Germplasm Resources in Technical System of Traditional Chinese Medicine Industry in Hebei Province (HBCT201806201), China Agriculture Research System (CARS21), and Technical Innovation Program of Hubei Province (2019ZYYD064). The funders had no role in the design of the study, data collection, analysis and interpretation, decision to publish, or preparation of the manuscript.

\section{Availability of data and materials}

All relevant supporting data sets are included in the article and its supplemental files.

\section{Ethics approval and consent to participate}

Not applicable.

\section{Consent for publication}

Not applicable.

\section{Competing interests}

The authors declare that they have no conflict of interest.

\section{Author details}

${ }^{1}$ Institute of Cash Crops, Hebei Academy of Agricultural and Forestry Sciences, Shijiazhuang 050051, Hebei, China. ${ }^{2}$ Institute of Chinese Herbal Medicines, Hubei Academy of Agricultural Sciences, Enshi 445000, Hubei, China.

Received: 22 April 2020 Accepted: 15 July 2020

Published online: 23 July 2020

\section{References}

1. Zhou L, Zuo Z, Chow MS. Danshen: an overview of its chemistry, pharmacology, pharmacokinetics, and clinical use. J Clin Pharmacol. 2005;45: $1345-59$.

2. Zhang $X Z$, Qian SS, Zhang YJ, Wang RQ. S. miltiorrhiza: a source for anti -Alzheimer's disease drugs. Pharm Biol. 2016;54(1):18-24.

3. Park B, Song HS, Kwon JE, Cho SM, Jang SA, Kim MY, et al. Effects of S. miltiorrhiza extract with supplemental liquefied calcium on osteoporosis in calcium-deficient ovariectomized mice. BMC Complement Altern Med. 2017;17(1):545.

4. Li YL, Xin XM, Chan ZY, Shi RJ, Miao ZM, Ding J, et al. The endophytic fungi of S. miltiorrhiza Bge.F. alba Are a potential source of natural antioxidants. Bot Stud. 2015;56:5.

5. He J, Giusti MM. Anthocyanins: natural colorants with health-promoting properties. Annu Rev Food Sci Technol. 2010;1:163-87. 
6. Jaakola L. New insights into the regulation of anthocyanin biosynthesis in fruits. Trends Plant Sci. 2013;18:477-83.

7. Quattrocchio F, Baudry A, Lepiniec L, Grotewold E. The regulation of flavonoid biosynthesis. Sci Flavonoids. 2006. https://doi.org/10.1007/978-0 $-387-28822-2$ _4

8. Falcone Ferreyra ML, Rius SP, Casati P. Flavonoids: biosynthesis, biological functions, and biotechnological applications. Front Plant Sci. 2012. https:// doi.org/10.3389/fpls.2012.00222

9. Li S, Strid A. Anthocyanin accumulation and changes in CHS and PR-5 gene expression in Arabidopsis thaliana after removal of the inflorescence stem (decapitation). Plant Physiol Biochem. 2005;43(6):521-5.

10. Cai $X$, Lin L, Wang $X, X u C$, Wang Q. Higher anthocyanin accumulation associated with higher transcription levels of anthocyanin biosynthesis genes in spinach. Genome. 2018;61(7):487-96.

11. Duan HR, Wang LR, Cui GX, Zhou XH, Duan XR, Yang HS. Identification of the regulatory networks and hub genes controlling alfalfa floral pigmentation variation using RNA-sequencing analysis. BMC Plant Biol. 2020;20:110.

12. Xie XB, Li S, Zhang RF, Zhao J, Chen YC, Zhao Q, et al. The bHLH transcription factor MdbHLH3 promotes anthocyanin accumulation and fruit colouration in response to low temperature in apples. Plant Cell Environ. 2012;35(11):1884-97.

13. Saito K, Yonekura-Sakakibara K, Nakabayashi R, Higashi Y, Yamazaki M, Tohge $T$, et al. The flavonoid biosynthetic pathway in Arabidopsis: structural and genetic diversity. Plant Physiol Biol. 2013;72:21-34.

14. Han Y, Vimolmangkang S, Soria-Guerra RE, Korban SS. Introduction of apple ANR genes into tobacco inhibits expression of both $\mathrm{CHI}$ and DFR genes in flowers, leading to loss of anthocyanin. J Exp Bot. 2012;63(7):2437-47.

15. Gonzalez A, Zhao M, Leavitt JM, Lloyd AM. Regulation of the anthocyanin biosynthetic pathway by the $T \mathrm{G} 1 / \mathrm{bHLH} /$ Myb transcriptional complex in Arabidopsis seedlings. Plant J. 2008:53:814-27.

16. Bai SL, Tao RY, Yin L, Ni JB, Yang QS, Yan XH, et al. Two B-box proteins, PpBBX18 and PpBBX21, antagonistically regulate anthocyanin biosynthesis via competitive association with Pyrus pyrifolia ELONGATED HYPOCOTYL 5 in the peel of pear fruit. The Plant J. 2019;100(6):1208-23.

17. Chen KL, Du L, Liu HL, Liu YL. A novel R2R3-MYB from grape hyacinth, MaMybA, which is different from MaAN2, confers intense and magenta anthocyanin pigmentation in tobacco. BMC Plant Biol. 2019;19:390.

18. Wu Q, Wu J, Li SS, Zhang HJ, Feng CY, Yin DD, et al. Transcriptome sequencing and metabolite analysis for revealing the blue flower formation in waterlily. BMC Genomics. 2016;17:897.

19. Lou Q, Liu Y, Qi Y, Jiao S, Tian F, Jiang L, et al. Transcriptome sequencing and metabolite analysis reveals the role of delphinidin metabolism in flower colour in grape hyacinth. J Exp Bot. 2014;65(12):3157-64.

20. Lei ZX, Zhou CH, Ji XY, Wei G, Huang YC, Yu WX, et al. Transcriptome analysis reveals genes involved in flavonoid biosynthesis and accumulation in Dendrobium catenatum from different locations. Sci Rep. 2018. https://doi. org/10.1038/s41598-018-24751-y.

21. Zhu G, Wang S, Huang Z, Zhang S, Lia Q, Zhang C, et al. Rewiring of the fruit metabolome in tomato breeding. Cell. 2018;172:249-61.

22. Paolo B, Saverio O, Mirko M, Matteo B, Lara G, Azeddine SA. Gene expression and metabolite accumulation during strawberry (Fragariaxananassa) fruit development and ripening. Planta. 2018;248(5):1143-57.

23. Dong T, Han R, Yu J, Zhu M, Zhang Y, Gong Y, et al. Anthocyanin accumulation and molecular analysis of correlated genes by metabolome and transcriptome in green and purple asparaguses (Asparagus officinalis, L). Food Chem. 2019;271:18-28.

24. Meng J, Wang B, He G, Wang Y, Tang XF, Wang SM, et al. Metabolomics integrated with transcriptomics reveals redirection of the phenylpropanoids metabolic flux in Ginkgo biloba. J Agric Food Chem. 2019;67(11):3284-91.

25. Li D, Chen G, Ma B, Zhong CG, He NJ. Metabolic profiling and transcriptome analysis of mulberry leaves provide insights into flavonoid biosynthesis. J Agric Food Chem. 2020;68:1494-504.

26. Petroni $\mathrm{K}$, Tonelli $\mathrm{C}$. Recent advances on the regulation of anthocyanin synthesis in reproductive organs. Plant Sci. 2011;181:219-29.

27. Lipka V, Kwon C, Panstruga R. SNARE-ware: the role of SNARE-domain proteins in plant biology. Annu Rev Cell Dev Bi. 2007;23:147-74.

28. Zhao D, Tao J, Han C, Ge J. Flower color diversity revealed by differential expression of flavonoid biosynthetic genes and flavonoid accumulation in herbaceous peony (Paeonia lactiflora pall.). Mol Biol Rep. 2012;39(12):11263-75.

29. Zhou TS, Wang XC, Yu YB, Xiao Y, Qian WJ, Xiao B, et al. Correlation analysis between total catechins (or anthocyanins) and expression levels of genes involved in flavonoids biosynthesis in tea plant with purple leaf. Acta Agron Sin. 2016:42(4):525.
30. Quintana A, Freyre R, Davis TM, Grisbach R. Genetic studies of flower color in Anagallis monelli L. Hortsci. 2008;43(6):1680-5.

31. Ben Simhon Z, Judeinstein S, Trainin T, Harel Beja R, Bar Ya'akov I, Borochov Neori $\mathrm{H}$, et al. A "white" anthocyanin-less pomegranate (Punica granatum L. ) caused by an insertion in the coding region of the leucoanthocyanidin dioxygenase (LDOX; ANS) gene. PLoS One. 2015;10(11):e0142777.

32. Bogs J, Jaffe FW, Takos AM, Walker AR, Robinson SP. The grapevine transcription factor VVMYBPA1 regulates proanthocyanidin synthesis during fruit development. Plant Physiol. 2007;143:1347-61.

33. Clark ST, Verwoerd WS. A systems approach to identifying correlated gene targets for the loss of colour pigmentation in plants. BMC Bioinformatics. 2011;12:343.

34. Debes MA, Arias ME, Grellet-Bournonville CF, Wulff AF, Martínez Zamora MG, Castagnaro AP, et al. White-fruited Duchesnea Indica (Rosaceae) is impaired in ANS gene expression. Am J Bot. 2011;98(12):2077-83.

35. Shimada S, Inoue YT, Sakuta M. Anthocyanidin synthase in nonanthocyanin-producing Caryophyllales species. Plant J. 2005;44(6):950-9.

36. Li HY, Liu JL, Pei TL, Bai ZQ, Han RL, Liang ZS. Overexpression of SmANS enhances anthocyanin accumulation and alters phenolic acids content in Salvia miltiorrhiza and Salvia miltiorrhiza Bge F. alba plantlets. Int J Mol Sci. 2019;20(9):2225.

37. Terrier N, Torregrosa L, Ageorges A, Vialet S, Verries C, Cheynier V, et al. Ectopic expression of VMMybPA2 promotes proanthocyanidin biosynthesis in grapevine and suggests additional targets in the pathway. Plant Physiol. 2008;149(2):1028-41.

38. Lloyd A, Brockman A, Aguirre L, Campbell A, Bean A, Cantero A, et al. Advances in the MYB-bHLH-WD repeat (MBW) pigment regulatory model: addition of a WRKY factor and co-option of an anthocyanin MYB for betalain regulation. Plant Cell Physiol. 2017;58(9):1431-41.

39. Solfanelli C, Poggi C, Loreti E, Alpi A, Perata P. Sucrose-specific induction of the anthocyanin biosynthetic pathway in Arabidopsis. Plant Physiol. 2006;140:637-46.

40. Hichri I, Barrieu F, Bogs J, Kappel C, Delrot S, Lauvergeat V. Recent advances in the transcriptional regulation of the flavonoid biosynthetic pathway. J Exp Bot. 2011;62(8):2465-83.

41. Chiu LW, Li L. Characterization of the regulatory network of BoMYB2 in controlling anthocyanin biosynthesis in purple cauliflower. Planta. 2012;36(4):1153-64.

42. Shi H, Liu G, Wei Y, Chan Z. The zinc-finger transcription factor ZAT6 is essential for hydrogen peroxide induction of anthocyanin synthesis in Arabidopsis. Plant Mol Biol. 2018;97(1-2):165-76.

43. Shi MZ, Xie DY. Biosynthesis and metabolic engineering of anthocyanins in Arabidopsis thaliana. Recent Pat Biotechnol. 2014;8:47-60.

44. Wang N, Liu W, Zhang T, Jiang S, Xu H, Wang Y, et al. Transcriptomic analysis of red-fleshed apples reveals the novel role of MdWRKY11 in flavonoid and anthocyanin biosynthesis. J Agric Food Chem. 2018;6(27):7076-86.

45. Lalusin A, Ocampo E, Fujimura T. Arabidopsis thaliana plants overexpressing the IbMADS10 gene from sweetpotato accumulates high level of anthocyanin. Philipp J Crop Sci. 2011;36(2):30-6.

46. Zhang M, Chen HX, Li JL, Pei Y, Liang Y. Antioxidant properties of tartary buckwheat extracts as affected by different thermal processing methods. Lwt-food Sci Technol. 2010;43(1):181-5.

47. Meng XC, Zhang YJ, Wang XQ. Content changes of anthocyanin, reducing sugar and soluble protein during the flower development of Petunia Hybrida. J South China Normal Univ. 2001;2:96-9.

48. Chen W, Gong L, Guo Z, Wang W, Zhang H, Liu X, et al. A novel integrated method for large-scale detection, identification, and quantification of widely targeted metabolites: application in the study of rice metabolomics. Mol Plant. 2013;6:1769-80.

49. Wang ZR, Cui YY, Vainstein A, Chen SW, Ma HQ. Regulation of fig (Ficus carica L.) fruit color: Metabolomic and transcriptomic analyses of the flavonoid biosynthetic pathway. Front Plant Sci. 2017. https://doi.org/10.3389/fpls.2017.01990.

50. Li JJ, Jiang CR, Brown JB, Huang H, Bickel PJ. Sparse linear modeling of next-generation mRNA sequencing (RNA-Seq) data for isoform discovery and abundance estimation. P Natl Acad Sci USA. 2011;108:19867-72.

51. Kanehisa M, Araki M, Goto S, Hattori M, Hirakawa M, Itoh $M$, et al. KEGG for linking genomes to life and the environment. Nucleic Acids Res. 2007;36:480-4.

52. Mao X, Cai T, Olyarchuk JG, Wei L. Automated genome annotation and pathway identification using the KEGG Orthology (KO) as a controlled vocabulary. Bioinformatics. 2005;21:3787-93.

53. Schmittgen TD, Livak KJ. Analyzing real-time PCR data by the comparative CT method. Nat Protoc. 2008:3:1101-8.

\section{Publisher's Note}

Springer Nature remains neutral with regard to jurisdictional claims in published maps and institutional affiliations. 